\section{Promoting pain resolution}

\section{By Michael J. Haas, Senior Writer}

Harvard Medical School researchers have shown that resolvins could help treat inflammatory pain without the side effects associated with opioids and COX-2 inhibitors. ${ }^{1}$ The findings could open up a new indication for Resolvyx Pharmaceuticals Inc., which is developing resolvins to treat inflammatory indications.

Charles N. Serhan, professor of anesthesiology at the medical school, led a research group that discovered resolvins in 2000 and showed they are a class of endogenous lipid mediators that promote the resolution of various inflammatory conditions, including sepsis.

Serhan is also director of the Center of Experimental Therapeutics and Reperfusion Injury at Brigham and Women's Hospital and a cofounder of Resolvyx.

Given the anti-inflammatory action of resolvins, "it was not difficult to guess that resolvins may work for inflammatory pain," said Ru-Rong Ji, who coled the current team with Serhan. "We also wanted to know if there were receptors for resolvins in neurons" in addition to their known targets on immune cells.

Ji is an assistant professor of anesthesiology at Brigham and Women's.

In several mouse models of acute and chronic inflammatory pain, nanogram injections of resolvin E1 (RvE1) or resolvin D1 (RvD1) into the spinal column or inflamed paw reduced pain, hyperalgesia and mechanical allodynia at least as effectively as microgram doses of morphine or a cyclooxygenase-2 (COX-2) inhibitor.

The resolvins did not affect normal responses to pain, which are important for protecting the body from potentially damaging stimuli.

The team also showed that RvE1 targeted both peripheral and central nervous system pathways involved in pain, including CNS neurons that express chemokine-like receptor 1 (CMKLR1; ChemR23), which RvE1 also targets on immune cells.

Data were reported in Nature Medicine.

Because the team demonstrated the effect of resolvins at both the molecular and behavioral levels, "from a preclinical proof-of-concept perspective there really isn't more one could do to show the role of resolvins in treating inflammatory pain," said Alasdair Naylor, CSO of Solace Pharmaceuticals Inc.
"Another advantage is that the team studied resolvins in models of inflammatory pain," which historically have been more representative of what happens in humans than have animal models of neuropathic pain, said Solace CEO Eliot Forster.

Solace has an inhibitor of sepiapterin reductase (SPR) in lead development for neuropathic pain and an inhibitor of GTP cyclohydrolase 1 (GCH1) in discovery for inflammatory pain. Both enzymes are involved in the biosynthesis of the pain-exacerbating molecule tetrahydrobiopterin (BH4).

"Any time you can open a window on a new mechanism to treat pain without side effects, it's exciting," said Daniel Carr, CMO of Javelin Pharmaceuticals Inc. "I certainly have the impression that this could develop into a therapy because of the new mechanism and the intrinsic margin of safety" implied by the lack of side effects in the mice.

Javelin's Dyloject diclofenac, an i.v. NSAID that inhibits cyclooxygenases (COX), is marketed in the U.K. to manage acute moderate to severe pain and is under FDA review for the same indication. Javelin has agreed to be acquired by Hospira Inc.

\section{Indications to resolve}

In the pain space, resolvins could have both safety and efficacy advantages over opioids and COX-2 inhibitors. On the efficacy front, resolvins actively promote the resolution of inflammatory pain instead of merely blocking the processes that sustain it.

At the same time, Naylor said, "one would expect resolvins to have fewer side effects than nonendogenous compounds that block proinflammatory responses" such as COX-2 inhibitors because resolvins act on a normal physiological pathway that resolves pain.

Carr agreed, noting that studies have shown that chronic use of NSAIDs can actually interfere with the resolution of pain.

Moreover, according to Resolvyx CSO Philip Vickers, "resolvins inhibit inflammation without causing immune suppression or interfering with the ability to fight infection." Thus, he said, resolvins could be used to treat painful inflammatory conditions associated with infection, such as post-herpetic neuralgia.

Vickers said the Nature Medicine paper also showed that resolvins might be "expected to provide important symptomatic benefit in inflammatory conditions that we are currently pursuing, including arthritis."

Resolvyx's lead compound RX-10045, a synthetic analog of RvE1 formulated into a topical eye drop, has completed Phase I/II testing to treat dry eye. RX-10001, an oral solution of synthetic RvE1, has completed Phase I testing to treat arthritis, asthma and inflammatory bowel disease (IBD).

The company also has resolvin analogs in discovery for undisclosed indications.

Both Carr and Forster agreed the anti-inflammatory and anti- 


\section{ANALYSIS}

\section{TARGETS \& MECHANISMS}

nociceptive effects of resolvins made them good candidates to treat rheumatoid arthritis (RA) and osteoarthritis (OA).

Carr also suggested the clinical opportunity would be enlarged because "shifting the focus of therapies from inhibition of proinflammatory mechanisms to the promotion of resolution mechanisms might be applicable to a larger group of patients," such as those with postherpetic neuralgia, persistent postoperative pain and complex regional pain syndrome.

Carr did say that resolvins are unlikely to replace NSAIDs, especially for the treatment of acute pain and types of chronic pain in which inhibiting the ongoing production of proinflammatory molecules would be an appropriate strategy.

He noted the benefits of NSAIDs are thought to outweigh their risks when given at the lowest effective dosage and for the minimum duration. "But you could imagine coadministering resolvins and NSAIDs to reduce the ongoing production of proinflammatory mediators and to accelerate resolution," he said.

Merouane Bencherif, VP of preclinical research at Targacept Inc., agreed.

"Pain and inflammation feed on each other so there is a need to affect both," Bencherif said. "That's the rationale for combining analgesic and anti-inflammatory agents, such as opioids and COX-2 inhibitors."

Targacept is developing nicotinic receptor antagonists to treat a range of neurological conditions, including pain, major depressive dis- order (MDD), attention deficit hyperactivity disorder (ADHD), cognitive dysfunction in schizophrenia and Alzheimer's disease (AD).

Ji said the team's ongoing work includes testing RvE1 and RvD1 in models of chronic, established neuropathic pain. The team is also investigating whether RvE1 and RvD1 work better in combination than either molecule alone, he said.

According to Serhan, Brigham and Women's has applied for a patent on the findings reported in Nature Medicine. The IP is unlicensed, although Resolvyx has in-licensed previous IP owned by Brigham and Women's that covers the use of resolvins to treat inflammatory indications.

Haas, M.J. SciBX 3(16); doi:10.1038/scibx.2010.483

Published online April 22, 2010

REFERENCES

1. Xu, Z.-Z. et al. Nat. Med.; published online April 11, 2010; doi: $10.1038 / \mathrm{nm} .2123$

Contact: Ru-Rong Ji, Brigham and Women's Hospital and Harvard Medical School, Boston, Mass.

e-mail: rrij@zeus.bwh.harvard.edu

Contact: Charles N. Serhan, same affiliation as above e-mail: cnserhan@zeus.bwh.harvard.edu

COMPANIES AND INSTITUTIONS MENTIONED

Brigham and Women's Hospital, Boston, Mass.

Harvard Medical School, Boston, Mass.

Hospira Inc. (NYSE:HSP), Lake Forest, III.

Javelin Pharmaceuticals Inc. (NYSE-A:JAV), Cambridge, Mass.

Resolvyx Pharmaceuticals Inc., Bedford, Mass.

Solace Pharmaceuticals Inc., Boston, Mass.

Targacept Inc. (NASDAQ:TRGT), Winston-Salem, N.C. 